\title{
Phlebographic Study Does Not Show Differences Between Patients with MS and Control Subjects
}

\author{
M. Stefanini, S. Fabiano, F. Garaci, S. Marziali, A. Meschini, V. Cama, M. Fornari, S. Rossi, D. Centonze, R. Gandini,
}

G. Simonetti, and R. Floris

\begin{abstract}
BACKGROUND AND PURPOSE: Hypothetical correlation between chronic cerebrospinal venous insufficiency and MS has gained the attention of patients and the scientific community. Studies performed by echo-color Doppler ultrasonography have shown different results, and it is necessary to use more objective diagnostic techniques. The aim of our study was to evaluate the presence of stenoses affecting azygos veins and internal jugular veins by use of venography in patients with MS.
\end{abstract}

MATERIALS AND METHODS: We recruited 2 groups of subjects who underwent venography: the study group included 29 patients with MS and the control group included 15 healthy volunteers. The ileo-lumbar plexus, the azygos, and the internal jugular veins were selectively catheterized. We considered any cross-sectional area reduction of the venous lumen $>50 \%$ to be a significant stenosis. Furthermore, blood pressure was measured in the studied vessels at the stenotic internal jugular veins.

RESULTS: Selective venography showed at least 1 significant venous stenosis in $84 \%$ of subjects examined, without significant difference between the study group and the control group. Positive venography chronic cerebrospinal venous insufficiency patterns were found in $50 \%$ of all subjects examined, without any significant difference between the 2 groups. The multivariate logistic regression analysis failed to assess any significant association between the presence of a positive venography and MS condition. The difference between the median blood pressure of stenotic and nonstenotic internal jugular veins was not statistically significant $(P=.46)$.

CONCLUSIONS: Our data exclude any direct correlation between chronic cerebrospinal venous insufficiency and MS because venous abnormalities were equally present in both groups.

ABBREVIATIONS: $A$ V = azygos vein; $C C S V I=$ chronic cerebrospinal venous insufficiency; $E D S S=$ Expanded Disability Status Scale; IJV = internal jugular vein; ILP = ileo-lumbar plexus

C hronic cerebrospinal venous insufficiency (CCSVI) is a nosological hypothesis that was discussed for the first time by the World Consensus Conference on Venous Malformations in Monte Carlo in September 2009. The CCSVI hypothesis suggests that inadequate blood flow from the CNS is caused by stenotic and/or malformative alterations of the main vessels draining blood from the brain and spine such as internal jugular veins (IJVs) and the azygos vein (AV). In this hypothesis, venous outflow is delayed or stopped; thus, toxic catabolites and iron cannot be removed and their deposition in the cerebral tissue is facili-

Received July 28, 2013; accepted after revision September 23.

From the Departments of Diagnostic Imaging (M.S., S.F., F.G., S.M., A.M., V.C., M.F., R.F., R.G., G.S.) and Neuroscience (S.R., D.C.), University of Rome Tor Vergata, Rome, Italy; and Santa Lucia Foundation/European Center for Brain Research (CERC) (S.R., D.C.), Rome, Italy.

Please address correspondence to Sebastiano Fabiano, MD, Department of Diagnostic Imaging, University of Rome Tor Vergata, Viale Oxford 81, 00133 Rome, Italy; e-mail: sebas575@yahoo.it

http://dx.doi.org/10.3174/ajnr.A3816 tated. This may cause a harmful effect, which may activate inflammatory response either in a direct and indirect way, leading to neuronal and oligodendrocytic damage.

MS is a chronic disease affecting the CNS with both neurodegeneration and inflammatory demyelination processes. The pathogenetic contribution of parenchymal iron deposition through proinflammatory action and oxidative damage has been hypothesized. ${ }^{1-3}$

A highly significant correlation between CCSVI and MS has been reported by Zamboni et $\mathrm{al}^{4}$ in a cohort of patients evaluated by echo-color Doppler ultrasonography.

Moreover, Zamboni et $\mathrm{al}^{5}$ led a pilot study enrolling $65 \mathrm{pa}-$ tients to assess the efficacy of percutaneous transluminal angioplasty of stenotic IJV and AV in the treatment of CCSVI. The authors of this study stated that the percutaneous treatment positively influenced the clinical condition and the quality of life of patients with MS when compared with the preoperative assessment.

At this time, several scientific endeavors examining the pres- 
Table 1: Demographic and clinical characteristics

\begin{tabular}{lccc}
\hline & $\begin{array}{c}\text { Study } \\
\text { Group }\end{array}$ & $\begin{array}{c}\text { Control } \\
\text { Group }\end{array}$ & P Value \\
\hline No. & 29 & 15 & \\
Sex, M/F & $10 / 19$ & $11 / 4$ & .03 \\
Age, $y$ & $46.4 \pm 9.5$ & $42.6 \pm 7.1$ & .18 \\
Disease duration, y & $12.6 \pm 6.7$ & NA & \\
Disease course (RR/SP/PP) & $17 / 8 / 4$ & NA & \\
EDSS & $3.6 \pm 2.1(1.0-7.0)$ & NA & \\
\hline
\end{tabular}

Note:-NA indicates not applicable; RR, relapsing-remitting; SP, secondary-progressive; PP, primary-progressive.

ence of CCSVI in MS are being undertaken, and other authors believe it is better for the health of patients to discourage potentially dangerous endovascular procedures until the question has been definitively clarified. ${ }^{6}$

Controversy exists to rule out whether the morphologic variability that occurs in the jugular veins should be considered a pathologic aspect or a normal anatomic variation, without any clinical consequences.

The aim of our study was to evaluate the presence of abnormalities and malformations affecting the IJV and AV by use of venography in patients with MS and to compare these findings with those from a control group of healthy volunteers.

\section{MATERIALS AND METHODS}

\section{Subjects and Clinical Assessments}

Between October 2011 and March 2012, we recruited 2 groups of subjects who underwent selective venography to evaluate the presence of morphologic and hemodynamic abnormalities involving the venous system. The study was approved by our institution's ethical committee, and all subjects signed a written informed consent for the procedure and personal data treatment.

The study group included 29 patients with MS free of relapse or steroid treatment in the 30 days before the study entry; the control group included 15 healthy volunteers who had no medical history of cerebrovascular or neurologic disorders.

The selection criteria used were age between $18-65$ years, normal renal function, and diagnosis of MS according to $2005 \mathrm{Mc}$ Donald Criteria. $^{7}$

Exclusion criteria for both groups were pre-existing medical conditions known to be associated with neck pathology, history of cerebral congenital vascular malformations, cerebral venous thrombosis, and previous selective venous catheterization. Family members of patients with MS were not considered eligible as control subjects.

Demographic and clinical data of enrolled subjects are presented in Table 1.

Demographic and clinical information of patients with MS was derived from medical records. MS disease onset was defined as the first episode of focal neurologic dysfunction indicative of MS. Disease duration was estimated as the number of years from onset to the last assessment of disability. Disability was determined by a specially trained and certified examining neurologist by use of the Expanded Disability Status Scale (EDSS), a 10-point disease severity score derived from 9 ratings for individual neurologic domains. ${ }^{8}$

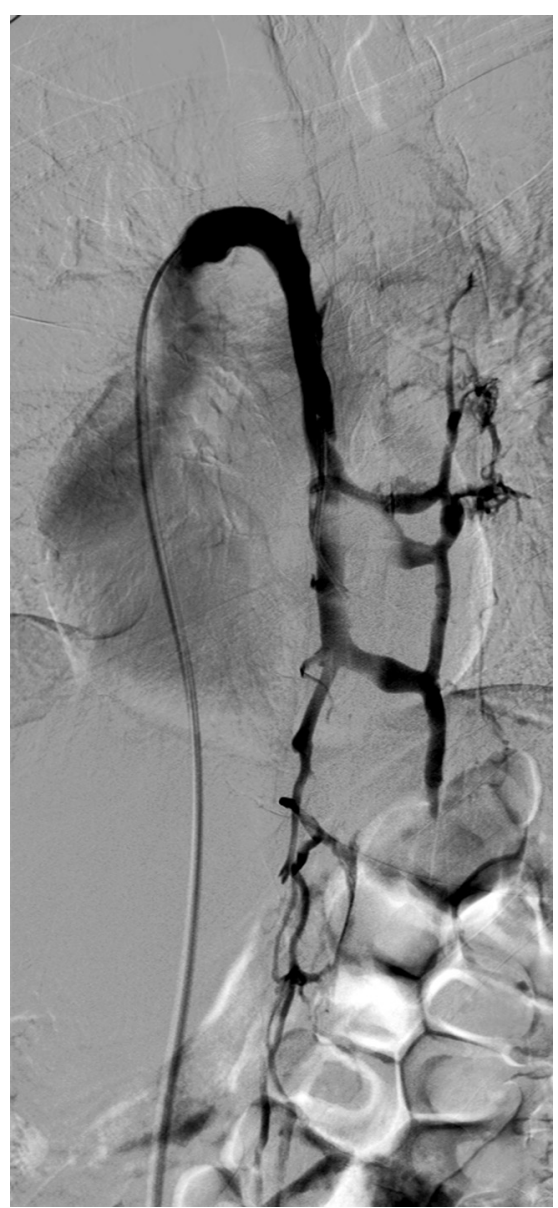

FIG 1. Selective catheterization of the azygos vein in a 37-year-old patient with secondary-progressive MS in which the injection of contrast medium showed the presence of a twisting of the $\mathrm{AV}$ associated with a shunt with the hemiazygous venous system. Note the opacification of the intraspinal venous plexus.

\section{Phlebographic Procedures}

All procedures were carried out in an angiographic suite by 2 interventional radiologists, a nurse, and an anesthesiologist. The patient was placed in the supine position; after a percutaneous transfemoral venous access was performed and a $5 \mathrm{~F}$ sheath was introduced, the ileo-lumbar plexus (ILP), the AV, and the IJV were selectively catheterized by use of either a Vertebral or a Cobra $15 \mathrm{~F}$ catheter (Terumo Europe, Leuven, Belgium). Contrast media was then injected at $3 \mathrm{~mL} / \mathrm{s}$ at the origin of the ILP $(10 \mathrm{~mL}$ of contrast media with the tip of the catheter at the level of the L5 vertebral body), at the confluence between the $\mathrm{AV}(10 \mathrm{~mL}$ of contrast) and the hemiazygous vein, and at the J3 level of the IJV corresponding to the upper part of the vein ( $10 \mathrm{~mL}$ of contrast media). The IJV was evaluated in the anterior-posterior oblique position to confirm the presence of alteration, whereas the AV was evaluated in the right posterior oblique projection (range, $10-15^{\circ}$ ). A 10-second phlebographic acquisition at 0.5 frames per second was performed with the patient holding his or her breath after a forced inspiration. In all patients, to confirm a normal contrast media outflow, we also performed a fluoroscopic visualization with the patient breathing normally. Furthermore, blood pressure was measured in the studied vessels at the J1 segment of the 

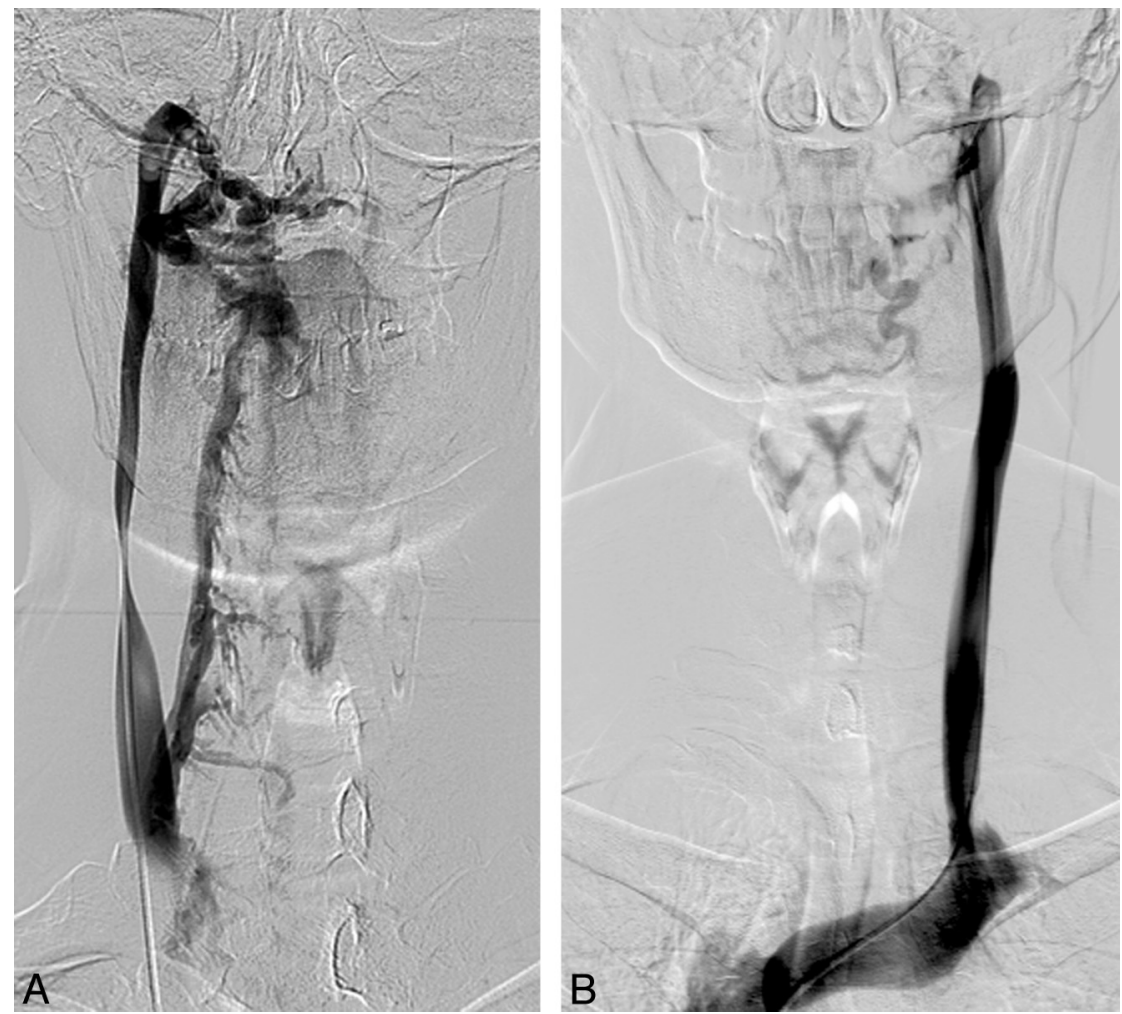

FIG 2. Selective catheterization of the left and right internal jugular veins in a 35-year-old patient with relapsing-remitting MS. Venous stenosis at the level of the section J2 of the right IJV and of its origin is shown, with associated drainage in spinal venous plexus through a collateral branch. The left IJV does not present alterations in size and drainage.

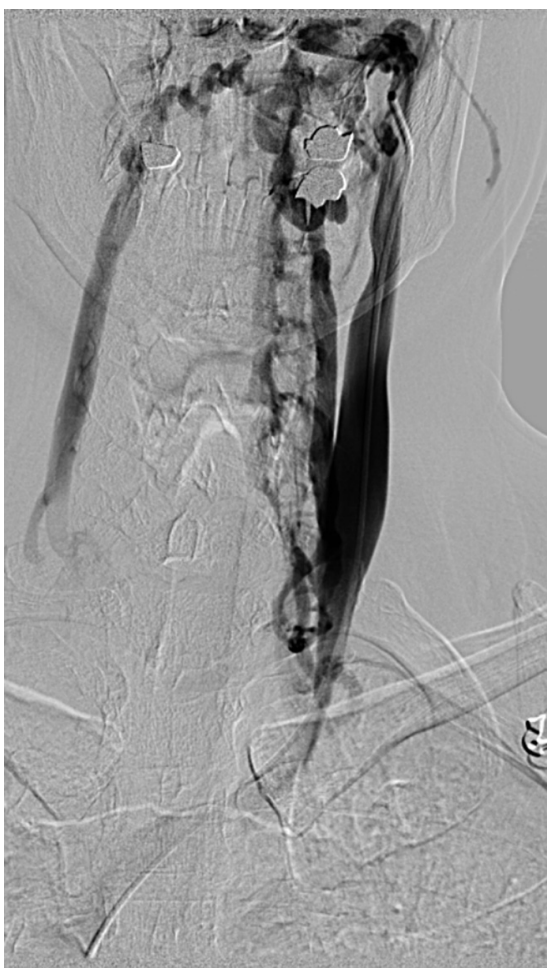

FIG 3. Selective catheterization of the left internal jugular vein in a 41-year-old patient with primary-progressive MS. After injection of contrast medium, stenosis of segment $\mathrm{J} 1$ with reflux in both vertebral veins and opacification of the vertebral venous plexus is documented.
IJV, brachiocephalic trunk, superior vena cava, inferior vena cava, and transstenoses by use of a PrimeWire Prestige Pressure Guide Wire (Volcano Corporation, San Diego, California).

At the end of the procedure, manual compression was performed, and all patients were discharged at 4 hours after an ultrasonography evaluation of the puncture site.

In accordance with Zamboni et $\mathrm{al},{ }^{5}$ we considered any cross-sectional area reduction of the venous lumen $>50 \%$ to be a significant stenosis. We have also evaluated the presence of vascular malformations at the level of the AV and the IJV, such as twisting, septum, annulus, and atresia. Thus, we referred to the patterns of Zamboni et al to classify our findings and to consider as positive for the further analysis:

Type A was classified as a steno-obstruction of the proximal AV, associated with a closed stenosis of 1 of the 2 IJVs.

Type B was classified as significant stenoses of both IJVs and the proximal AV.

Type C was classified as bilateral stenosis in both IJVs, with a normal AV.

Type D was classified as multiple ab-

normalities of the AV.

\section{Statistical Analysis}

Clinical and demographic data are expressed, unless otherwise specified, as mean \pm standard deviation. Comparisons between 2 groups of data were performed by the Student $t$ test or by the Mann-Whitney test. The Fisher exact test was used for contingency analyses. The MS condition was analyzed as the dependent variable in logistic regressions with sex and age and either the presence of a positive venography or the presence of at least 1 significant stenosis as predictors. The associations between either the presence of a positive venography or the presence of at least 1 significant stenosis and disease severity were assessed by use of multivariate binary logistic regression models with dichotomous EDSS (cutoff point of 4.0) or progressive status (categorizing relapsing-remitting MS as nonprogressive) as outcome variables. The significance level was set at $P<.05$.

\section{RESULTS}

Selective venography showed at least 1 significant extracranial venous stenosis in $84 \%$ of all subjects examined (37/44), without any significant difference $(P>.5)$ between the study group (25/ $29)$ and the control group (12/15).

Stenosis or malformations of the AV were observed in 9 subjects (Fig 1). Those of the right IJV were found in 19 subjects (Fig 2), stenosis or malformations of the left IJV in 17 subjects (Fig 3), and those of the ILP in 15 subjects (Fig 4).

Regression analysis confirmed the lack of association between 


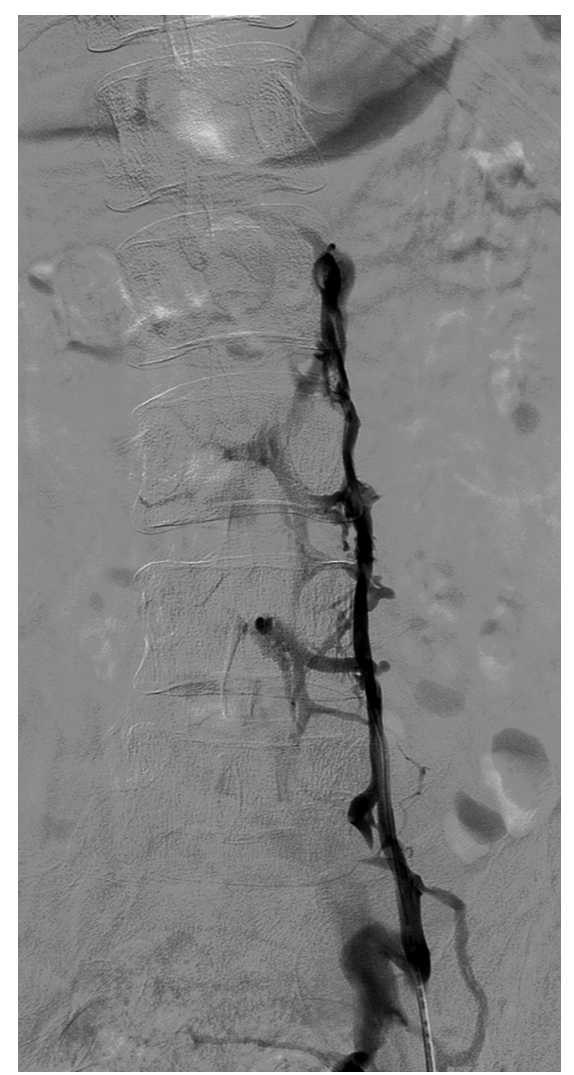

FIG 4. Selective catheterization of the ileo-lumbar plexus in a 33year-old patient affected by relapsing-remitting MS. After injection of the contrast medium, opacification of the intraspinal venous plexus without opacification of the inferior vena cava is documented.

Table 2: Logistic regression (MS condition as outcome variable)

\begin{tabular}{lccccc}
\hline & Coefficient & SE & OR & \multicolumn{1}{c}{$95 \% \mathrm{Cl}$} & $\boldsymbol{P}$ Value \\
\hline Venography & 0.77 & 1.24 & 2.16 & $0.18-24.91$ & .53 \\
Stenosis, n & -0.01 & 0.59 & 0.98 & $0.30-3.19$ & .98 \\
Age & 0.06 & 0.04 & 1.06 & $0.97-1.15$ & .16 \\
Sex, male & -1.57 & 0.72 & 0.20 & $0.05-0.86$ & .03 \\
\hline
\end{tabular}

Note:-SE indicates standard error.

the presence of MS condition and at least 1 significant venous stenosis $(\mathrm{OR}=1.12 ; 95 \% \mathrm{CI}, 0.17-7.07 ; P=.89)$, correcting for age and sex.

Positive venography patterns, as described by Zamboni et al,, ,5 were found in $50 \%$ of all subjects examined (22/44), without any significant difference $(P=.5)$ between the study group (16/29) and the control group (6/15). In line with this, the multivariate logistic regression analysis, also including the number of significant stenoses as an independent variable, failed to assess any significant association between the presence of a positive venography and MS condition (Table 2).

Furthermore, type $\mathrm{C}$ was the more frequent hemodynamic pattern observed among positive subjects, without significant differences between study and control groups (10/16 versus $4 / 6, P>$ $.5)$, as shown in Table 3 , in which the 4 hemodynamic patterns were also stratified according to MS disease course.

We then assessed the association between the venographic findings and measures of disease severity among the MS group. The presence of at least 1 significant stenosis failed to predict progressive status, correcting for age and sex $(\mathrm{OR}=1.97 ; 95 \% \mathrm{CI}$,
Table 3: Venographic pattern distribution

\begin{tabular}{cccccc}
\hline Type & Study Group & Control Group & RR MS & SP MS & PP MS \\
\hline A & 4 & 2 & 3 & 1 & 0 \\
B & 1 & 0 & 0 & 1 & 0 \\
C & 10 & 4 & 8 & 1 & 1 \\
D & 1 & 0 & 0 & 1 & 0 \\
\hline
\end{tabular}

Note:- RR indicates relapsing-remitting; SP, secondary-progressive; PP, primary-progressive.

Table 4: Logistic regression (progressive MS as outcome variable)

\begin{tabular}{lccccc}
\hline & Coefficient & SE & OR & 95\% Cl & P Value \\
\hline Venography & -2.26 & 1.60 & 0.10 & $0.00-2.38$ & .15 \\
Stenosis, $n$ & 0.76 & 0.76 & 2.14 & $0.48-9.56$ & .31 \\
Age & 0.10 & 0.05 & 1.12 & $1.00-1.24$ & .04 \\
Sex, male & 0.83 & 0.92 & 2.29 & $0.37-14.19$ & .37 \\
\hline
\end{tabular}

Note:-SE indicates standard error.

$0.14-27.83 ; P=.61)$. In the multivariate logistic regression analysis, including the presence of a positive venography and the number of significant stenoses among the independent variables, the only significant predictor of progressive status was age (Table 4 ). Furthermore, the probability of EDSS $\geq 4.0$ was associated neither with the presence of at least 1 venous stenosis $(\mathrm{OR}=0.48$; 95\% CI, $0.03-7.11 ; P=.59)$ nor with a positive venography $(\mathrm{OR}=0.14 ; 95 \% \mathrm{CI}, 0.00-3.36 ; P=.22)$ in logistic regressions correcting for age, sex, and disease duration. Finally, neither the number of venous stenoses nor the probability of having a positive venography differed significantly in patients treated with different immunomodulator agents during 2 years before the study entry or in never-treated patients (not shown).

The difference between the median blood pressure of stenotic IJVs ( $13.59 \mathrm{~cm} \mathrm{H}_{2} \mathrm{O}$; range, 40.79-28.50 $\mathrm{cm} \mathrm{H}_{2} \mathrm{O}$ ) and nonstenotic IJVs ( $16.314 \mathrm{~cm} \mathrm{H}_{2} \mathrm{O}$; range, $8.57-28.55 \mathrm{~cm} \mathrm{H}_{2} \mathrm{O}$ ) was not statistically significant $(P=.46)$.

The difference between the mean blood pressure of stenotic AVs (19.03 $\mathrm{cmH}_{2} \mathrm{O}$; range, $\left.10.87-27.19 \mathrm{~cm} \mathrm{H}_{2} \mathrm{O}\right)$ and nonstenotic AVs (18.35 cm $\mathrm{H}_{2} \mathrm{O}$; range, 9.51-38.06 $\mathrm{cm} \mathrm{H}_{2} \mathrm{O}$ ) was not statistically significant $(P=.79)$.

The mean procedural time was 28 minutes (range, 22-43 minutes).

No major or minor procedural and postprocedural complications were recorded, and patients were discharged 4 hours after the end of the percutaneous procedure.

\section{DISCUSSION}

CCSVI is a nosological hypothesis recently defined and characterized by abnormalities of the venous system of the neck and thorax causing defective blood drainage from the CNS. ${ }^{4,5}$

The recent description of CCSVI and its hypothesized strong correlation with MS has gained the attention of both the scientific community and patients, especially in consideration of the potential reversibility of this condition. This correlation appears to be confirmed by the studies of Zamboni et al, ${ }^{5,9}$ who not only reported the presence of CCSVI in all patients affected by MS but also published a study on the feasibility of the endovascular treatment of this condition and its positive effects on MS progression. Despite several reports supporting this hypothesis, ${ }^{10-14}$ in recent literature we found studies reporting disagreeing data and, in some cases, doubting the existence of CCSVI itself, all on the basis of echo-color Doppler ultrasonography evaluation., ${ }^{2,15-20}$

AJNR Am J Neuroradiol 35:1174-79 Jun 2014 www.ajnr.org 


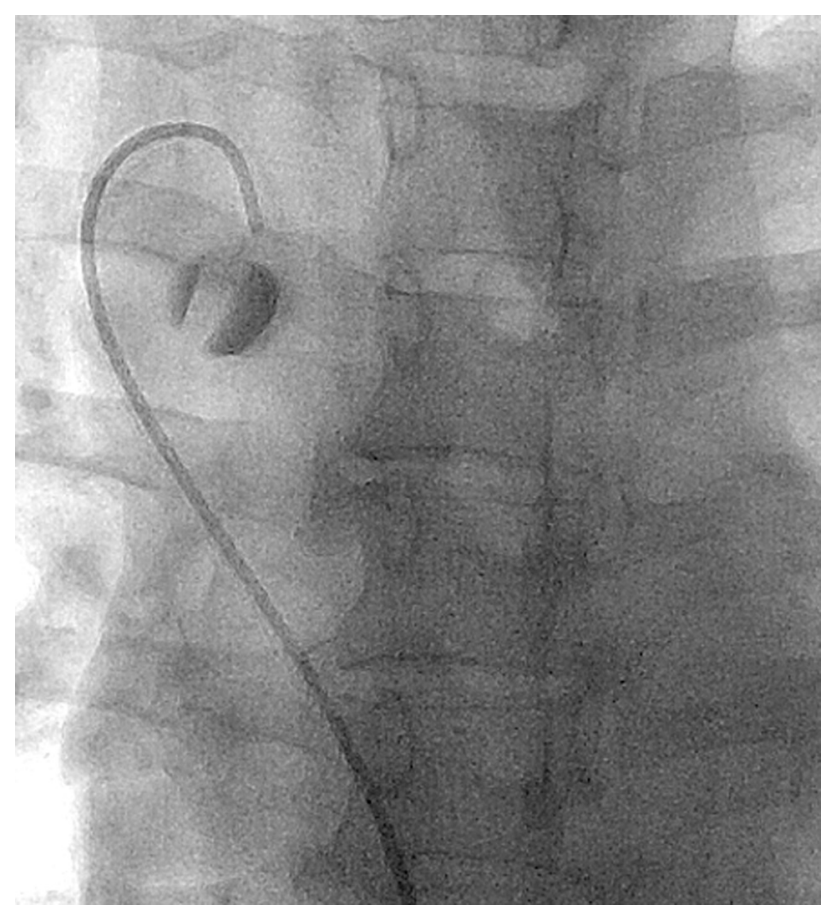

FIG 5. Selective catheterization of the azygos vein in a 45-year-old patient affected by relapsing-remitting MS. At the confluence of the azygos vein after administration of contrast medium, a valve is evident.
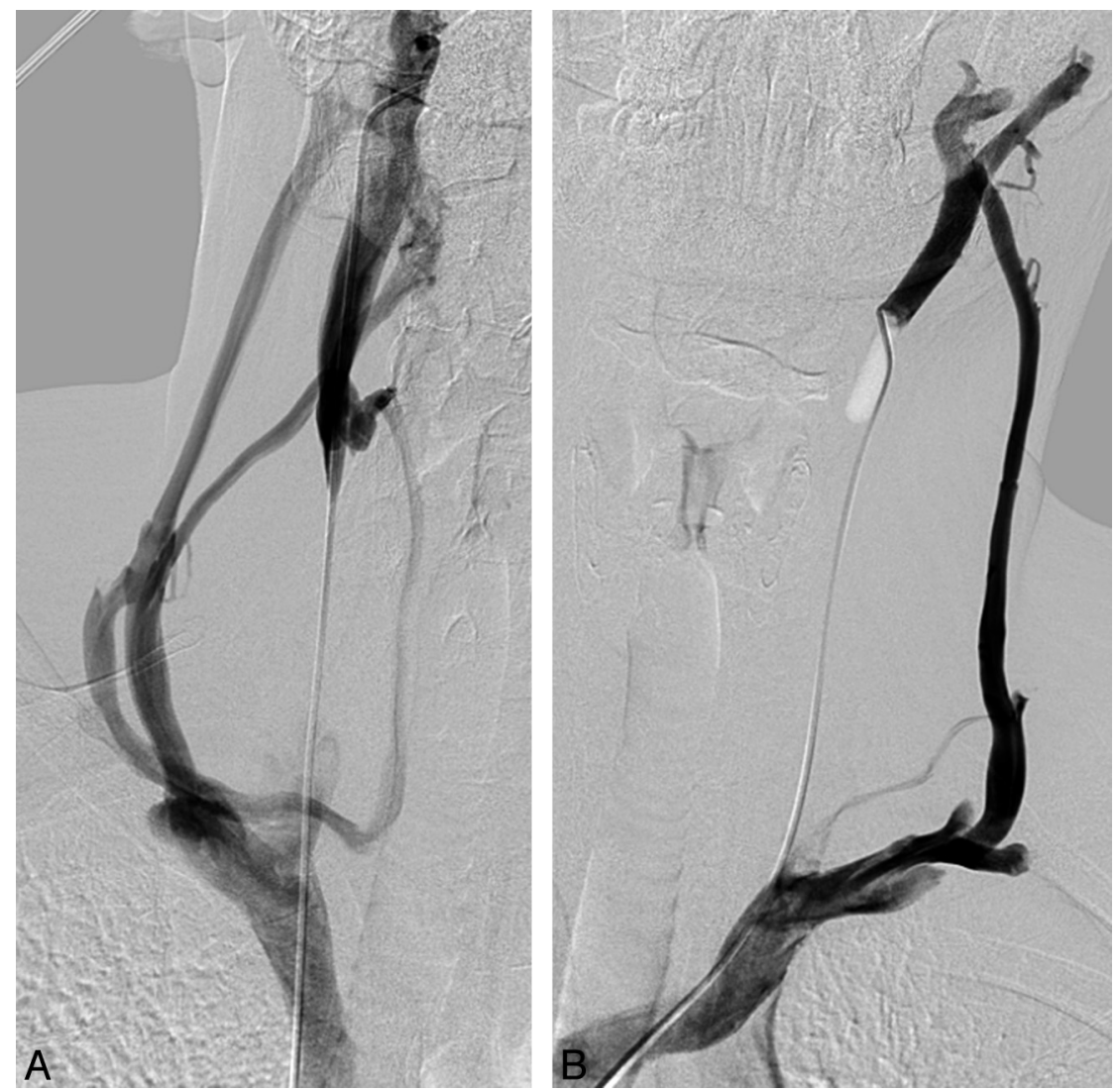

FIG 6. Selective catheterization of the internal jugular veins bilaterally $(A,-B)$ in a 27 -year-old control subject. Complete obstruction of the IJVs bilaterally with evidence of venous outflow through collateral circulation in the external jugular veins is shown.
Even recent studies, on the basis of MR imaging, ${ }^{21-23}$ do not appear to demonstrate the association. The choice of a diagnostic method with high sensitivity and specificity is a key point. Nowadays, the most reliable techniques are compared with each other. ${ }^{24}$

We therefore decided to investigate the CCSVI hypothesis by use of venography, which is considered to be the reference standard ${ }^{24}$ in evaluation of the venous system. The results of our study show significant discrepancies from those reported by Zamboni et al in terms of stenosis of the ILP (34\% in our experience versus $9 \%$ of Zamboni et $\left.\mathrm{al}^{4}\right)$ and of the AV (20.5\% versus $\left.71 \%\right)$. Regarding the latter, the difference may be due to the fact that despite the evidence of what is defined by Zamboni et al as a "membranous obstruction at the junction of the AV with the superior vena cava" present in all patients (Fig 5), such finding never correlated with a reduced venous outflow or a significant abnormality in terms of intravenous pressure. Furthermore, these so-called "obstructions" were never associated with rachidian collaterals. In fact, AV arch valves have been reported to be common and should not necessarily be interpreted as pathologic findings. ${ }^{25}$

In $95 \%$ of the patients, IJV stenoses were found just above the termination of the IJVs. However, a reduced caliber of the vessel at such level was present in all patients and in all cases, and it did not correlate with a significant blood pressure gradient as reported by Zamboni et al. Because it has been demonstrated that the presence of jugular valves is very frequent, the reported reduction of caliber and/or stenosis of the IJVs, in the absence of collateral drainage vessels, probably should not be interpreted as a pathologic finding but as a physiologic condition caused by the valve's fibrous ring and contiguous anatomic structures (the collar bone and the sternocleidomastoid muscle).

Given our data, we believe that with venography it is not possible to define a pathologic condition characterized by the presence of a reduced blood drainage from the CNS as the result of stenotic alterations or venous malformations of the neck and thorax, which definitely correlates with MS. In our opinion, 1 of the major limitations of venography in the evaluation of CCSVI may be the lack of standardization in the interpretation of the images, which therefore tends to be operator-dependent, especially when no blood pressure gradient is associated with morphologic alterations. In our series, only 6 patients presented with the complete occlusion of 1 or both IJVs with the evidence of voluminous collateral drainage (Fig 6), and in only 1 case the stenosis of the AV was associated with rachidian drainage. However in all of these cases, no significant blood pressure gradient was recorded. In the remainder of our population, the interpretation of venograms could have been influenced not only by the operator's experience but also several parameters of contrast media injection 
(volume, length, and site of injection) as well as patient breath dynamics.

We found no significant difference in terms of incidence of CCSVI between subjects with MS and control subjects; moreover, in both groups, the presence of at least 1 stenosis was higher, suggesting that these alterations are present in general population without a higher incidence in patients with MS. No significant association of single patterns of Zamboni et al venographic classification was found. Furthermore, considering single venographic type and MS forms, we are in line with Zamboni et al regarding the distribution of Type A (more frequent among relapsing-remitting MS), but we observed a different distribution of the other types.

The absence of any statistically significant correlations between extracranial venous stenosis and MS or disability progression may suggest that these alterations are unrelated to MS pathogenesis. This study, although with the statistical limitations of a small sample size, is the first to assess the relationship between CCSVI condition and MS, performing selective venography in both patients with MS and healthy volunteers.

\section{CONCLUSIONS}

Our data exclude any direct correlation between CCSVI and MS because venous stenosis was equally present in both groups. However, the possibility that they play a role in other pathologies cannot be excluded. Indeed, further studies are needed to standardize the evaluation of CCSVI, obtaining images from broader populations of patients and larger control groups, to assess definitive venographic features of this proposed clinical entity.

Disclosures: Dr Silvia Rossi received honoraria for writing from Bayer Schering and funding for traveling from Novartis, Teva, Merck Serono. She acted as an Advisory Board member of Biogen Idec and is involved as sub-investigator in clinical trials for Novartis, Merck Serono, Teva, Bayer Schering, Sanofi-Aventis, Biogen Idec, Roche. Disclosures not included above (ICMJE): UNRELATED: Payment for Lectures (including service on speakers bureaus): Novartis; Payment for Manuscript Preparation: Bayer; Travel/Accommodations/Meeting Expenses Unrelated to Activities Listed: Biogen. Dr Diego Centonze acted as an Advisory Board member of Merck-Serono, Teva, Bayer Schering, Biogen Idec, Novartis, Almirall, and received funding for traveling and honoraria for speaking or consultation fees from Merck Serono, Teva, Novartis, Bayer Schering, Sanofi-Aventis, Biogen Idec. He is the principal investigator in clinical trials for Novartis, Merck Serono, Teva, Bayer Schering, Sanofi-Aventis, Biogen Idec, Roche. Disclosures not included above (ICMJE): UNRELATED: Board Membership: GW Pharmaceuticals, Fondazione Italiana Sclerosi Multipla, Comments: Including prior board membership disclosures, €20,000; Consultancy: Teva, Merck Serono, Biogen, Novartis, Genzyme, Comments: €10,000; Grants/Grants Pending: Fondazione Italiana Sclerosi Multipla, ${ }^{*}$ Comments: $€ 400,000$; Payment for Lectures (including service on speakers bureaus): Teva, Merck Serono, Biogen, Novartis, Genzyme, Allmiral GW, Comments: $€ 6000$.

\section{REFERENCES}

1. Singh AV, Zamboni P. Anomalous venous blood flow and iron deposition in multiple sclerosis. J Cereb Blood Flow Metab 2009;29:1867-78

2. Worthington V, Killestein J, Eikelenboom MJ, et al. Normal CSF ferritin levels in MS suggest against etiologic role of chronic venous insufficiency. Neurology 2010;75:1617-22

3. Williams R, Buchheit CL, Berman NE, et al. Pathogenic implications of iron accumulation in multiple sclerosis. $J$ Neurochem 2012;120:7-25

4. Zamboni P, Galeotti R, Menegatti E, et al. Chronic cerebrospinal venous insufficiency in patients with multiple sclerosis. J Neurol Neurosurg Psychiatry 2009;80:392-99

5. Zamboni P, Galeotti R, Menegatti E, et al. A prospective open-label study of endovascular treatment of chronic cerebrospinal venous insufficiency. J Vasc Surg 2009;50:1348-58

6. Khan O, Filippi M, Freedman MS, et al. Chronic cerebrospinal venous insufficiency and multiple sclerosis. Ann Neurol 2010;67:286-89

7. Polman CH, Reingold SC, Edan G, et al. Diagnostic criteria for multiple sclerosis: 2005 revisions to the "McDonald Criteria." Ann Neurol 2005;58:840-46

8. Kurtzke JF. Rating neurologic impairment in multiple sclerosis: an expanded disability status scale (EDSS). Neurology 1983;33:1444-52

9. Malagoni AM, Galeotti R, Menegatti E, et al. Is chronic fatigue the symptom of venous insufficiency associated with multiple sclerosis? A longitudinal pilot study. Int Angiol 2010;29:176-82

10. Al-Omari MH, Rousan LA. Internal jugular vein morphology and hemodynamics in patients with multiple sclerosis. Int Angiol 2010;29:115-20

11. Beggs C. Multiple sclerosis appears to be associated with cerebral venous abnormalities. Ann Neurol 2010;68:560-61

12. Hojnacki D, Zamboni P, Lopez-Soriano A, et al. Use of neck magnetic resonance venography, Doppler sonography and selective venography for diagnosis of chronic cerebrospinal venous insufficiency: a pilot study in multiple sclerosis patients and healthy controls Int Angiol 2010;29:127-39

13. Simka M, Zaniewski M. Reinterpreting the magnetic resonance signs of hemodynamic impairment in the brains of multiple sclerosis patients from the perspective of a recent discovery of outflow block in the extracranial veins. J Neurosci Res 2010;88:1841-45

14. Zivadinov R, Schirda C, Dwyer MG, et al. Chronic cerebrospinal venous insufficiency and iron deposition on susceptibilityweighted imaging in patients with multiple sclerosis: a pilot casecontrol study. Int Angiol 2010;29:158-75

15. Doepp F, Paul F, Valdueza JM, et al. No cerebrocervical venous congestion in patients with multiple sclerosis. Ann Neurol 2010;68: 173-83

16. Centonze D, Floris R, Stefanini M, et al. Proposed chronic cerebrospinal venous insufficiency criteria do not predict multiple sclerosis risk of severity. Ann Neurol 2011;70:51-58

17. Garaci F, Marziali S, Meschini A, et al. Brain hemodynamic changes associated with chronic cerebrospinal venous insufficiency are not specific to multiple sclerosis and do not increase its severity. Radiology 2012;265:233-39

18. Floris R, Centonze D, Fabiano S, et al. Prevalence study of chronic cerebrospinal venous insufficiency in patients with multiple sclerosis: preliminary data. Radiol Med 2012;117:855-64

19. Mayer CA, Pfeilschifter W, Lorenz MW, et al. The perfect crime? CCSVI not leaving a trace in MS. J Neurol Neurosurg Psychiatry 2011;82:436-40

20. Valdueza JM, Doepp F, Schreiber SJ, et al. What went wrong? The flawed concept of cerebrospinal venous insufficiency. J Cereb Blood Flow Metab 2013;33:657-68

21. Sundström P, Wåhlin A, Ambarki K, et al. Venous and cerebrospinal fluid flow in multiple sclerosis: a case-control study. Ann Neurol 2010;68:255-59

22. Wattjes MP, van Oosten BW, de Graaf WL, et al. No association of abnormal cranial venous drainage with multiple sclerosis: a magnetic resonance venography and flow-quantification study. J Neurol Neurosurg Psychiatry 2011;82:429-35

23. Zivadinov R, Cutter G, Marr K, et al. No association between conventional brain $M R$ imaging and chronic cerebrospinal venous insufficiency in multiple sclerosis. AJNR Am J Neuroradiol 2012;33: 1913-17

24. Zaharchuk G, Fischbein NJ, Rosenberg J, et al. Comparison of MR and contrast venography of the cervical venous system in multiple sclerosis. AJNR Am J Neuroradiol 2011;32:1482-89

25. Yeh BM, Coakley FV, Sanchez HC, et al. Azygos arch valves: prevalence and appearance at contrast-enhanced CT. Radiology 2004;230: $111-15$ 